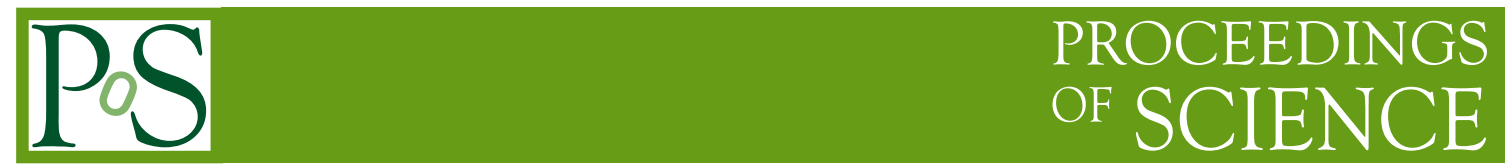

\title{
Search for Invisible Higgs in CMS
}

\author{
Sunil Bansal ${ }^{* 1}$, K. Mazumdar ${ }^{2}$ and J. B. Singh ${ }^{1}$ \\ ${ }^{1}$ Panjab University, Chandigarh, India \\ ${ }^{2}$ Tata Institute for Fundamental Research, Mumbai, India \\ E-mail: 9.bansal@gmail.com, Ka jari.Mazumdar@cern.ch, \\ Jasbir.Singh@cern.ch
}

\begin{abstract}
Discovery of the Higgs boson and the subsequent study of its properties is one of the main physics goals of the LHC. Invisible decay mode of the Higgs boson is an interesting possibility in several scenarios of Beyond Standard Model physics. The search strategy for an invisible decaying Higgs boson, produced in Vector Boson Fusion process, has been studied in detail, along with backgrounds using detailed simulation of the CMS experiment. In cut based analysis, QCD background is found to be overwhelming. With data corresponding to an integrated luminosity of 10 $\mathrm{fb}^{-1}$, an upper limit of $36 \%$ on the branching ratio of $H \rightarrow$ invisible can be explored for Higgs boson of mass $120 \mathrm{GeV} / \mathrm{c}^{2}$. The situation improves with more data and with $30 \mathrm{fb}^{-1}$ an upper limit as low as $21 \%$ can be probed.
\end{abstract}

XXth Hadron Collider Physics Symposium

November 16 - 20, 2009

Evian, France

*Speaker. 


\section{Introduction}

Most of the Higgs search analysis assume that the Higgs boson will predominantly decay into detectable particles. However, in Beyond Standard Model (BSM) scenarios, this may not be a good assumption if there are new weakly interacting particle, which couples to the Higgs boson with large enough strengths. In particular, if this weakly interacting particle are neutral and stable, the Higgs boson will decay invisibly. There are many models in which this situation is realized; e.g., when the Higgs boson can decay to a pair of lightest neutralinos in SUSY model with the unification constraint at GUT scale removed [1], 4th generation neutrino [2], Large Extra Dimensions [3] etc.

In the present analysis, the invisible Higgs boson is assumed to be produced with SM production rate and decaying with $100 \%$ branching fraction. Consequently, the results can be scaled for a non-SM Higgs boson production rate as well as partly-visible decay branching fractions.

\section{Analysis Strategy}

Since the Higgs boson decays invisibly, processes with additional particles in the final state have to be utilized. For this purpose, the Vector Boson Fusion (VBF) production mode of Higgs boson, qq $\rightarrow \mathrm{qqH}$, is the best suited, since the topological properties of the VBF events can be conveniently utilized to control backgrounds as pointed out in [4] and production rate is second highest over full range of Higgs boson mass. The main backgrounds for this search are $\mathrm{W}+$ jets, $\mathrm{Z}$ + jets and QCD multi-jet production which have large production rates at LHC.

The online and offline selections of signal events are mainly based on properties of VBF jets. The online selection or trigger requires the $E / \mathrm{T} \geq 60 \mathrm{GeV}$ and atleast 2 jets in the event satisfying the conditions:

$$
p_{T}^{j 1, j 2}>40 \mathrm{GeV}, \quad \eta_{j 1} \times \eta_{j 2}<0, \quad \text { and } \quad \Delta \eta_{j 1 . j 2}>4.2
$$

The trigger efficiency for signal in the mass range 120 to $140 \mathrm{GeV} / \mathrm{c}^{2}$ is about $18 \%$.

During off-line analysis, an event is rejected if it contains any isolated electron with $p_{T}>10$ $\mathrm{GeV} / c$ and a muon with $p_{T}>5 \mathrm{GeV} / c$. The leading two jets in the event are required to have $p_{T}>$ $40 \mathrm{GeV} / c$, in opposite hemispheres, i.e., $\eta_{j 1} \times \eta_{j 2}<0$. These jets are required to have substantial pseudo-rapidity gap: $\Delta \eta_{j 1, j 2}>4.4$ and reasonably large value of invariant mass: $M_{j 1, j 2}>1200$ $\mathrm{GeV} / c^{2}$. Events are further filtered with the condition of having $E /{ }_{\mathrm{T}}$ above $100 \mathrm{GeV}$.

Applying a veto on any additional hadronic activity between the two tagging jets is aimed to reduce effectively almost all the above backgrounds while keeping a high signal selection efficiency. In this Central Jet Veto (CJV) condition, an event with a 3rd jet is rejected if it has uncorrected transverse energy greater than a threshold of $15 \mathrm{GeV}$ and if it is located in the region defined by $\eta^{j \min }+0.5<\eta^{j 3}<\eta^{j \max }-0.5$ where $\eta^{j \min }$ or $\eta^{j \max }$ refers to the rapidities of the tagging jets and $\eta^{j 3}$ corresponds to the 3 rd jet.

To tackle mainly the QCD multi-jet backgrounds to a satisfactory level, a jet energy-based criteria is used based on the variable:

$$
N V=\frac{\left(E_{T}^{m i s s}\right)^{2}-\left(E_{T}^{j 1}-E_{T}^{j 2}\right)^{2}}{\left(E_{T}^{j 2}\right)^{2}}
$$


Ideally $N V$ will be zero for QCD di-jet events and positive for events which have real missing energy like in our case of signal events. If hard gluon radiation is pre-dominant in one of the jets, it will cause the quantity $E_{T}^{j 1}-E_{T}^{j 2}$ to be large. On the other hand, since $E /{ }_{\mathrm{T}}$ is measured taking into account all calorimeter towers (and consequently all jets), it will be less sensitive to this effect, and hence, leading to a negative $N V$ value. Finally, for the signal events, since the heavy Higgs boson is balanced by the two jets, the jets are expected to be acoplanar. Hence to discrimante signal vs. backgrounds, total number of events are counted in the range $\Delta \Phi_{j j} \leq 1.5 \mathrm{rad}$ and the signal yield is obtained by subtracting the expected background level in this region. Thus background rate estimation is very crucial for the analysis and should be maximally data-driven.

\subsection{Data Driven Analysis}

With few tens of $\mathrm{pb}^{-1}$ of data, the rates of $\mathrm{Z} / \mathrm{W}+n$-jets background events $(n=1,2,3, \ldots)$ are expected to be determined accurately. Further, the branching ratios of $\mathrm{Z}$ and $\mathrm{W}$ to charged and neutral lepton modes are well measured. Effectively, after accounting for the branching ratios, the difference between the two processes reduces to that due to detector acceptance. Subsequently, the fraction of $\mathrm{W} / \mathrm{Z}+$ jets events which are likely to pass the VBF criteria and other particular selections for our analysis can be obtained. The cross-section for $Z(\rightarrow v v)+$ jets mode can be estimated using both $\mathrm{Z}(\rightarrow \ell \ell)+$ jets as well as $\mathrm{W}(\rightarrow \ell v)+$ jets processes. For an integarted luminosity of $1 \mathrm{fb}^{-1}$ this background is estimated to contribute $63 \pm 10$ events using $\mathrm{W}(\mu v)+$ jets process which is quite good agreement with our results from simulated $Z \rightarrow v v+$ jets events, ie., $64 \pm 7$ events. The $\mathrm{W}+$ jets background where the charged lepton is not detected and hence contributes to $E / \mathrm{T}$, can be estimated using $\mathrm{W}+$ jets process itself where the lepton is well measured. For example, using this method for $\mathrm{W}(\rightarrow \mu v)+$ jets channel, the estimated number of $40 \pm 7$ events is in good agreement with the estimate of $43 \pm 7$ events using cut based analysis.

\section{Results}

For $1 \mathrm{fb}^{-1}$ of accumulated luminosity, the statistical significance of the signal, $S / \sqrt{B}$ value, is

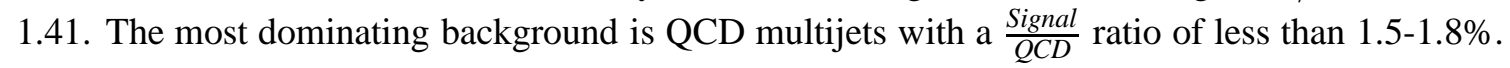
The uncertainty in the number of selected signal events is $14.5 \%$ due to uncertainty in jet energy scale (JES) and 9.5\% due to that in missing transverse energy scale. The results of the analysis for different values of Higgs boson mass is shown in Table 1.

In order to estimate the discovery potential, the best way is to provide the experimentally estimated number of signal events, in excess of backgrounds, which can be related to any scenario of BSM physics that envisages an invisibly decaying Higgs boson. For this purpose the model-independent variable $\xi^{2}$ is defined as:

$$
\xi^{2}=\mathrm{Br}(\mathrm{H} \rightarrow \text { Inv. }) \frac{\sigma_{\mathrm{BSM}}}{\sigma_{\mathrm{SM}}}
$$

Thus, if $\sigma_{\mathrm{BSM}}=\sigma_{\mathrm{SM}}$, then $\xi^{2}$ is equivalent to the invisible decay branching fraction $\mathrm{Br}$. The de-

\begin{tabular}{|l|c|c|c|}
\hline Higgs mass $\left(\mathrm{GeV} / \mathrm{c}^{2}\right)$ & 120 & 140 & 160 \\
\hline$\sigma(\mathrm{fb})$ & 4470 & 3830 & 3320 \\
\hline Trigger Eff.(\%) & 16.27 & 17.57 & 18.65 \\
\hline Offline Eff. (\%) & 15.30 & 14.99 & 14.42 \\
\hline Final $\sigma(\mathrm{fb})$ & 109 & 101 & 89 \\
\hline$S / \sqrt{B}$ & 1.41 & 1.30 & 1.15 \\
\hline
\end{tabular}

Table 1: The signal significance for different values of Higgs boson mass. The total number of background events is estimated to be about 6000 . 
pendence of $\xi^{2}$ parameter as a function of Higgs boson mass is shown in Fig 1 for different values of the integrated luminosity.

\section{Conclusions}

A strategy to search for the Higgs boson in CMS experiment in its invisible decay mode is investigated using VBF production mechanism at LHC centre of mass energy of $14 \mathrm{TeV}$. The event topology and other kinematic properties of signal events are utilized to tackle various Standard Model backgrounds. The data driven methods for estimation of $\mathrm{V}+$ jets background processes have been suggested, which yield satisfactory results within statistical uncertainty. To say anything about discovery or exclusion of this decay mode, understanding of QCD background is very crucial which is only possible with real data. With reduced energy of LHC, the trend of production rates of the signal and various backgrounds is different, and discovery potential remains to be estimated while waiting for sufficient real collision data.

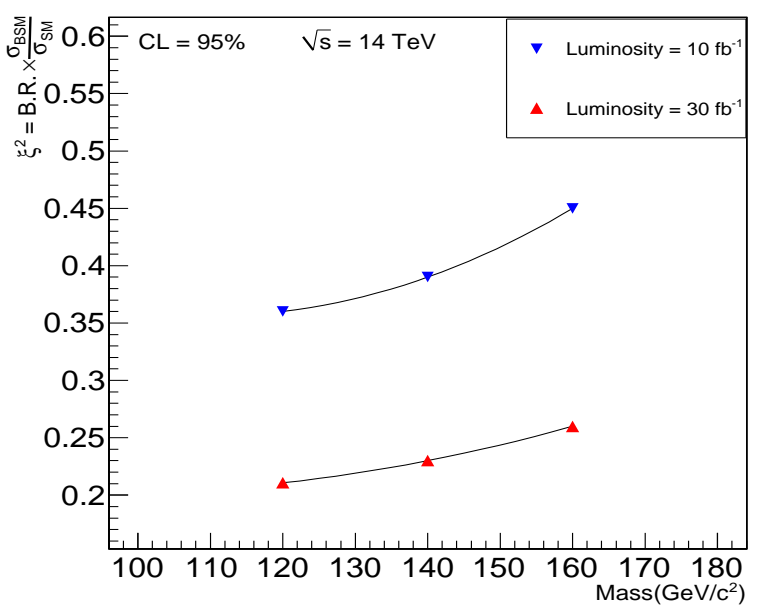

Figure 1: $\xi^{2}$ as function of Higgs boson mass $\left(\mathrm{GeV} / \mathrm{c}^{2}\right)$ for different values of integrated luminosity.

\section{Acknowledgment}

We sincerely thank organizers of symposium and CMS colloboation to provide the opportunity to present this work. S. Bansal will like to thank DST, DAE and CSIR-HRDG for funding.

\section{References}

[1] F. Boudjema, G. Belanger, R. M. Godbole, Invisible Decays of the Supersymmetric Higgs and Dark Matter, hep-ph/0206311.

[2] K. Belotsky, D. Fargion, M. Khlopov, R.Konoplich, K.Shibaev, Invisible Higgs Boson Decay into Massive Neutrinos of 4th Generation, hep-ph/0210153.

[3] D. Dominici, Probing Extra Dimensions through the Invisible Higgs Decay, hep-ph/0503216.

[4] O. J. P. Éboli and D. Zeppenfeld, Observing an Invisible Higgs Boson, Phys. Lett. B 495, 147 (2000). 